\title{
Comparative Study of the Cellular Uptake and Intracellular Behavior of a Library of Cyclic Peptide-Polymer Nanotubes with Different Self-Assembling Properties
}

Sean H. Ellacott, Carlos Sanchez-Cano, Edward D.H. Mansfield, Julia Y. Rho, Ji-Inn Song, Raoul Peltier, and Sébastien Perrier*

Cite This: Biomacromolecules 2021, 22, 710-722

Read Online

ACCESS | Lلll Metrics \& More | 回 Article Recommendations ｜ sl Supporting Information

ABSTRACT: Particle shape has been described as a key factor in improving cell internalization and biodistribution among the different properties investigated for drug-delivery systems. In particular, tubular structures have been identified as promising candidates for improving drug delivery. Here, we investigate the influence of different design elements of cyclic peptide-polymer nanotubes (CPNTs) on cellular uptake including the nature and length of the polymer and the cyclic peptide building block. By varying the composition of these cyclic peptide-polymer conjugates, a library of CPNTs of lengths varying from a few to over a $150 \mathrm{~nm}$ were synthesized and characterized using scattering techniques (small-angle neutron scattering and static light scattering). In vitro studies with fluorescently labeled CPNTs have shown that nanotubes comprised of a single polymer arm with a size between 8 and $16 \mathrm{~nm}$ were the most efficiently taken up by three different mammalian cell lines. A mechanistic study on multicellular tumor spheroids has confirmed the ability of these compounds to penetrate to their core. Variations in the proportion of paracellular and transcellular uptake with the self-assembling potential of the CPNT were also observed, giving key insights about the behavior of CPNTs in cellular systems.

\section{INTRODUCTION}

The design of novel nanomaterials for a diverse range of biological applications has gained great interest in recent years. The use of nanoparticle (NP) technologies, in particular for drug delivery, has allowed for enhanced bioavailability and increased efficacy of some drugs; as well as a reduction in the occurrence of side effects. ${ }^{1}$ Different types of nanomaterials of various morphologies have been investigated for these purposes (e.g.,: polymeric micelles, ${ }^{2}$ liposomes, ${ }^{3}$ and nanogels). ${ }^{4,5}$ Studying the stability and behavior of drug-free NP systems in vitro and in vivo is essential to develop more efficient and biocompatible systems. Cellular uptake of NPs usually occurs via different pathways (endocytic or not) and understanding this is of vital importance for drug-delivery systems. This process is known to be dependent on a variety of physicochemical properties, including surface charge, hydrophobicity, or the presence of targeting moieties, ${ }^{6}$ but is highly directed by the size and shape of the NPs. ${ }^{7,8}$ In particular, cylindrical or rod-like particles are known to have improved circulation times and cellular uptake when compared with their spherical counterparts. ${ }^{9}$ For example, it has been observed that dextran-coated magnetic iron nanoworms, both labeled or not labeled with a targeting peptide sequence, were more efficient at targeting tumors selectively in vivo than their spherical analogues. ${ }^{10}$ Changing the aspect ratio of cylindrical polymer brushes also enables these systems to be tailored to obtain improved uptake by tumors, in both spheroid and in vivo models. ${ }^{11,12}$ These changes in the cellular uptake efficiency between spheres and rods were attributed to differences in the membrane wrapping of the particles. Furthermore, computational studies demonstrated that the mode of entry differs with the aspect ratio and the general morphology of the nanostructure, also suggesting an orientation-dependence. ${ }^{13}$ The importance of shape on intracellular behavior was further highlighted in the work of Hinde et al. where polymerizationinduced self-assembly was employed to generate architectures of different sizes and shapes. The intranuclear uptake was studied by the authors using pair-correlation microscopy; it was found that despite adding a nuclear localization signal tag on spherical polymer NPs, passage through the nuclear pore complex was still less efficient than with untagged rods or worms. $^{14}$

Received: October 20, 2020

Revised: December 10, 2020

Published: December 22, 2020 
Two of the most common challenges with previously described systems is to ensure their biodegradability and clearance from the body. The use of self-assembling materials, based on supramolecular interactions, could be a way to circumvent this issue for drug delivery. ${ }^{15}$ Self-assembling organic nanotubes are then ideal systems for drug delivery, but also have potential applications in numerous fields, such as sensing, catalysis, and ion channel mimics. ${ }^{16}$ Amidst these selfassembling systems, cyclic octapeptides, comprising of alternating D- and L-amino-acids and able to self-assemble into tubular $\beta$-sheet-like structures, have been considered of interest for biological applications. ${ }^{17,18}$ These self-assemblies can lead to uncontrollable, insoluble aggregation; better control over the stacking process and improved solubility in water can be obtained through conjugation of hydrophilic polymers to the cyclic peptides (CP)..$^{19-21}$

The potential for cyclic peptide polymer nanotubes (CPNTs) in the delivery of anticancer drugs has previously been explored. Blunden et al. have found that in the case of antimetastatic $\mathrm{Ru}$-based drug, $\mathrm{RAPTA}-\mathrm{C}$, the $\mathrm{IC}_{50}$ was reduced by a factor of 18 on the A2780 ovarian cancer cell line when attached to a CPNT. ${ }^{22}$ More recently, the same anticancer activity was observed with a much more potent Ir complex. ${ }^{23}$ The $\mathrm{IC}_{50}$ value of the drug-bearing CPNT was lower than those of the free drug and polymer conjugate on ovarian cancer cell line A2780. A better specificity of the nanotubes toward cancer cells was also noticed, as the toxicity of the Ir-containing CPNT was six times lower on healthy ovarian cells than on A2780. Such changes were not correlated with a higher content of $\mathrm{Ir}$ in the cell, suggesting a more efficient mode of action for CPNTs, in comparison to the free drug and nonassembling polymer controls. ${ }^{22,23}$ An in vivo study looking at the pharmacokinetics and biodistribution of CPNTs also showed clear advantages of these systems over common NPs used: the possibility for prolonged blood circulation combined to an efficient excretion via disassembly. ${ }^{24}$ Despite these promising results, little is known on what design parameters are of importance to make optimal use of CPNTs for biological applications. Very different polymer CPNTs were considered in the aforementioned studies, hence the need for a more systematic study of the uptake of nondrug-containing CPNTs.

Herein, we report the synthesis of a library of different fluorescently labeled cyclic peptide-polymer conjugates. By varying the number of polymer chains, and the composition and morphology of the polymer used, we obtained systems with different self-assembling behavior. Their cellular uptake was then evaluated on $2 \mathrm{D}$ cell models to establish patterns related to the propensity of the conjugates for self-assembly. Diffusion phenomena and intracellular behavior were also investigated in $3 \mathrm{D}$ models with the help of confocal laser scanning microscopy (CLSM).

\section{EXPERIMENTAL (MATERIALS AND METHODS)}

Characterization of the Self-Assemblies. Small Angle Neutron Scattering. Small-angle neutron scattering (SANS) was performed on the SANS-2D instrument at the ISIS Pulsed Neutron Source (STFC Rutherford Appleton Laboratory, Didcot, U.K.). In a SANS experiment, the scattering from a collimated neutron beam is measured, from which a scattering cross section can be generated. By plotting the scattering intensity $I(q)$ as a function of the wave vector $q$, it is possible to establish the size and shape of the scattering species. Samples were left to dissolve in deuterated water for $16 \mathrm{~h}$ prior to measurement, at a concentration of 5 (for compounds 8, 9, and 10) or $2 \mathrm{mg} / \mathrm{mL}$ (for 6 and 7) and transferred into $2 \mathrm{~mm}$ quartz Hellma cuvettes. The $q$-range, where the scattering wave vector $q$ is defined as ( $q=\frac{4 \pi}{\lambda} \sin \frac{\theta}{2}$, with $\theta$ being the scattered angle and $\lambda$ the incident neutron wavelength) for all measurements was $0.0040-0.73 \AA^{-1}$. The beam diameter was $8 \mathrm{~mm}$, with an incident wavelength range varying between 1.75 and $16.5 \AA$. The small-angle detector was placed $4 \mathrm{~m}$ from the cuvette, offset vertically $60 \mathrm{~mm}$ and sideways $100 \mathrm{~mm}$, following a previous protocol established for the study of CPNTs. ${ }^{17}$ The temperature was set to $25{ }^{\circ} \mathrm{C}$. The raw data for each sample measurement were corrected for background, $\mathrm{D}_{2} \mathrm{O}$, detector efficiency, sample transmission, and empty cuvette and reduced using instrument software Mantid resulting in a scattering crosssection for each sample, and placed on an absolute scale using a perdeuterated polystyrene. ${ }^{25}$ The reduced data were fitted to different form factors using the SASfit software package. ${ }^{26}$ Details about the form factors used and the different fitting parameters are provided in the Supporting Information (section Characterization of the selfassemblies, Tables S3-S9).

Static Light Scattering. Light scattering measurements presented here were acquired on the ALV-CGS3 system (ALV-GmBH, Langen, Germany), with a vertically polarized laser source at $632 \mathrm{~nm}$. The measurements were taken at $25{ }^{\circ} \mathrm{C}$. Samples were prepared $16 \mathrm{~h}$ before measuring and filtered through a $200 \mu \mathrm{m}$ GHP filter (Acrodisc, $13 \mathrm{~mm}$ diameter, VWR International, United Kingdom) into clean cylindrical glass cuvettes (inner diameter: $8 \mathrm{~mm}$; outer diameter: 10 $\mathrm{mm}$; height: $75 \mathrm{~mm}$; LS Instruments, Switzerland); the $\mathrm{d} n / \mathrm{d} c$ was set at 0.1 during the measurement and corrected by its real value after measuring on a refractometer. The data were modeled to a Zimm equation, and Zimm plots were drawn. ${ }^{27}$ The molecular weight of the self-assemblies was then determined, and the number of aggregation was obtained after dividing it by the molecular weight of the unimer constituting the self-assembly. The plots are provided in Figure S17 in the Supporting Information.

Cellular Biology. Cell Culture. MDA-MB-231 HM cells (epithelial metastatic breast cancer cells; ECACC 92020424) and PC-3 (epithelial prostate cancer cells; ECACC 90112714) were grown in Dulbecco's modified Eagle's medium (DMEM) supplemented with $10 \%(\mathrm{v} / \mathrm{v})$ fetal bovine serum and $2 \mathrm{mM}$ of L-glutamine and $1 \%(\mathrm{v} / \mathrm{v})$ penicillin $\left(100 \mathrm{IU} \cdot \mathrm{mL}^{-1}\right) /$ streptomycin $\left(100 \mu \mathrm{g} \cdot \mathrm{mL}^{-1}\right)$ at $37{ }^{\circ} \mathrm{C}$ in a humid $5 \% \mathrm{CO}_{2}$ environment. Cells were typically passaged at $80-90 \%$ confluence. NIH $3 \mathrm{~T} 3$ cells (murine fibroblasts; ECACC 93061524) were grown in DMEM supplemented with $10 \%$ $(\mathrm{v} / \mathrm{v})$ bovine calf serum and $2 \mathrm{mM}$ of L-glutamine and $1 \%(\mathrm{v} / \mathrm{v})$ penicillin $\left(100 \mathrm{IU} \cdot \mathrm{mL}^{-1}\right) /$ streptomycin $\left(100 \mu \mathrm{g} \cdot \mathrm{mL}^{-1}\right)$ at $37^{\circ} \mathrm{C}$ in a humid $5 \% \mathrm{CO}_{2}$ environment. All cell lines were routinely screened for mycoplasma infection using the MycoAlert Mycoplasma Detection Kit (LT07-218; Lonza, US) as described in the instruction of the kit. No more than passage number 20 of each cell-line was used in this study.

Cytotoxicity Assay (XTT/PMS). Toxicity of the different nonfluorescent conjugates was assessed using a standard XTT protocol. The polymer and CP conjugates to be tested were dissolved in water with $0.5 \%$ dimethyl sulfoxide in order to obtain solutions at $500 \mu \mathrm{M}$. These solutions were subsequently used to prepare dilutions in a mixture of culture media (DMEM) and phosphate-buffered saline (PBS) (50:50) at the following concentrations: 100, 50, 10, 1, and 0.1 $\mu \mathrm{M}$. MDA-MB-231 cells were seeded in a transparent Greiner 96 well-plate at a density of 25,000 cells per well and incubated for $24 \mathrm{~h}$. The culture media was then removed, and subsequently replaced by $100 \mu \mathrm{L}$ of the prepared solutions. After $24 \mathrm{~h}$ incubation, the mixture was replaced with fresh media supplemented with $25 \mu \mathrm{L}$ of the XTT solution $\left(1 \mathrm{mg} \cdot \mathrm{mL}^{-1}\right)$ containing $N$-methyl dibenzopyrazine methyl sulfate (PMS) $\left(25 \mu \mathrm{mol} \cdot \mathrm{L}^{-1}\right)$. Cells were incubated for another $17 \mathrm{~h}$. Absorbance was then directly measured using a BioTek Cytation 3 Cell Imaging Multi-Mode Reader at 450 and $650 \mathrm{~nm}$ (background). The cell growth (in percentage) relative to untreated control cells was calculated using the following formula 
Scheme 1. Overview of the Different Fluorescent CP-Polymer Conjugates Investigated ${ }^{a}$

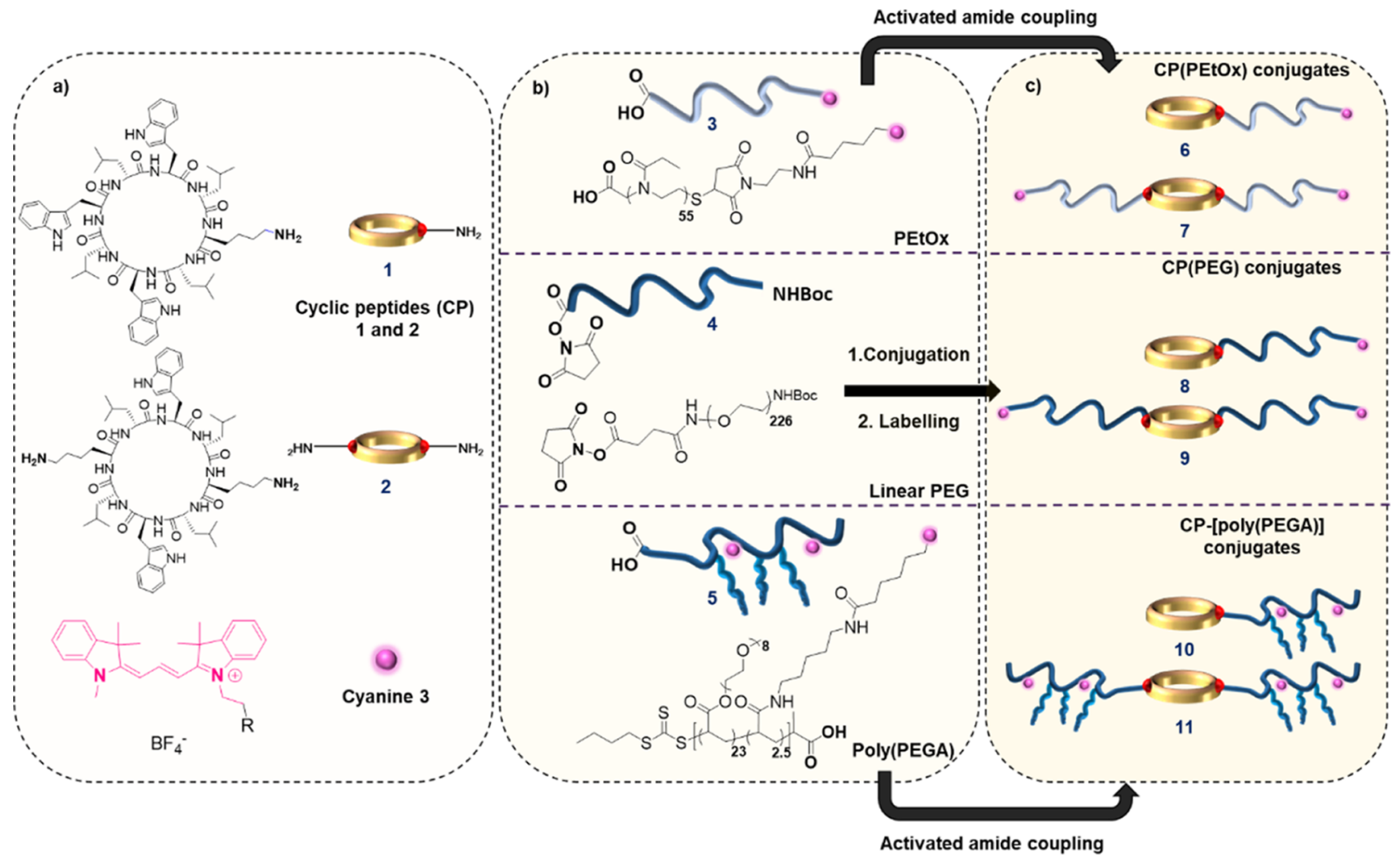

${ }^{a}$ (a) Cyclic peptides 1 and 2 and the dye molecule, cyanine 3, (b) different labeled hydrophilic polymers (3, 4, and 5), and (c) the final structures obtained by amide coupling onto cyclic peptides $(6,7,8,9,10$, and 11)

$$
\begin{aligned}
& \text { viability }(\%)= \\
& \qquad \begin{array}{r}
(\text { A450[experimental }]-\text { A450[blank }]- \text { A650[experimental }]) \\
(\text { A450[control }]-\text { A450[blank }]- \text { A650[control }]) \\
\times 100
\end{array}
\end{aligned}
$$

The viability data presented are the average of two repeats where each sample was measured in triplicate. Errors reported correspond to the standard deviation of the mean. The data are presented in Figure S18 in the Supporting Information.

Cell Uptake Study: Microplate Reading Experiment. Solutions of Cy3-labeled polymer and CP conjugates at 5 and $25 \mu \mathrm{M}$ were prepared in cell media. NIH 3 T3 or MDA-MB-231 cells were seeded in a transparent Greiner 96 well-plate at a density of 10,000 or 15,000 cells per well and incubated for $24 \mathrm{~h}$. The media were subsequently replaced by the prepared solutions of compounds and the cells were left incubating for a further 2 or $24 \mathrm{~h}$.

Hoechst 33342 (nuclei staining dye) was added and incubation proceeded for another $15 \mathrm{~min}$ before cells were washed with warm media twice. Cells were imaged in media using a BioTek Cytation 3 Cell Imaging Multimode Reader plate reader, set at absorption/ emission detection of 350/450 $\mathrm{nm}$ for Hoechst 33342 and 555/575 $\mathrm{nm}$ for Cy3 (RFP filter). The average Cy3 fluorescence emission per well was then calculated, setting a cut-off of the cell limitations from the nuclei and excluding all fractions of cells expressing saturation ( $>10,000$ A.U. of fluorescence intensity). The values were corrected using the values obtained from a fluorescence calibration study for all of the Cy3-labeled compounds. All data are reported as the means of two or three repeats, as bar charts with an overlap of all repeats. Error bars represent the standard error of the mean. Details of the correction factors for the processing of the images can be found in the Supporting Information (from Table S10).

Cell Uptake Study: Microplate Reading Experiment at $4{ }^{\circ} \mathrm{C}$. Solutions of Cy3-labeled polymer and CP conjugates at $12.5 \mu \mathrm{M}$ were prepared in cell media. PC-3 cells were seeded in a transparent Greiner 96-well plate at a density of 10,000 cells per well and incubated for $48 \mathrm{~h}$ with $150 \mu \mathrm{L}$ of cell media. For the $4{ }^{\circ} \mathrm{C}$ assay itself, the plate was preincubated at $4{ }^{\circ} \mathrm{C}$ for $30 \mathrm{~min}$ before replacing with $50 \mu \mathrm{L}$ of the prepared solutions of compounds and the cells were left incubating for a further $2 \mathrm{~h}$, at 37 or $4{ }^{\circ} \mathrm{C}$. Hoechst 33342 (nuclei staining dye) was added and incubation proceeded for another $15 \mathrm{~min}$ before cells were washed with warm or cold (for the $4{ }^{\circ} \mathrm{C}$ experiment) medium twice. $100 \mu \mathrm{L}$ of the fresh medium was eventually added. Cells were imaged using a BioTek Cytation 3 Cell Imaging Multimode Reader, set at absorption/emission detection of 350/ $450 \mathrm{~nm}$ for Hoechst 33342 and 555/575 nm for Cy3 (RFP filter).

The average $\mathrm{Cy} 3$ fluorescence emission per well was then calculated, setting a cut-off of the cell limitations from the nuclei and excluding all fractions of cells expressing saturation (>7000 A.U. of fluorescence intensity). The values were corrected using the values obtained from a fluorescence calibration study for all of the Cy3labeled compounds. All data are reported as the means of two biological replicates with error bars representing the standard error of the mean. Details of the processing of the images can be found in the Supporting Information.

Cell Uptake Study: Confocal Microscopy on 2D Cell Cultures. For confocal microscopy, PC-3 cells were seeded in a 10 -well plate at a density of 20,000 cells per well, and allowed to grow for $48 \mathrm{~h}$ prior to the experiment. Cell media were then replaced by fresh media supplemented with one of the four compounds of interest at $5 \mu \mathrm{M}$ from stock solutions at $500 \mu \mathrm{M}$ in water (two wells were used for each compound) and were incubated for $24 \mathrm{~h}$. LysoTracker Deep Red was added $2 \mathrm{~h}$ before imaging, Hoechst 33342 (nuclei stain) was then added and incubation proceeded for another $15 \mathrm{~min}$ before cells were washed with warm PBS twice. The cells were left in colorless media and imaged immediately. Confocal microscopy images were taken on a Leica TCS SP5 (Carl Zeiss, Germany) at a temperature of $37^{\circ} \mathrm{C}$, using a $\times 40$ objective and sequential scanning for each channel. 
Excitation/Emission procedure used for imaging is as follows: nucleus channel (405/406-459 nm), RFP channel for Cy3 compounds (561/ 557-598 nm), and LysoTracker Deep Red channel (633/646-698 $\mathrm{nm})$.

Spheroid Culture-Liquid Overlay Method. A suspension of PC-3 cells at a concentration of $10^{4}$ cells $/ \mathrm{mL}$ was prepared from a passage of $80-90 \%$ confluent PC- 3 cells. $200 \mu \mathrm{L}$ of cells $\left(2 \times 10^{3}\right.$ cells $)$ were seeded in a Cellstar Cell-Repellent Surface 96-well plates. The plate was subsequently centrifuged for $5 \mathrm{~min}$ at $500 \mathrm{~g}$ and left incubating for 4 days at $37{ }^{\circ} \mathrm{C}$ with $5 \% \mathrm{CO}_{2}$ to form spheroids.

Confocal Microscopy on Spheroids. PC-3 spheroids were grown following the liquid overlay method. $100 \mu \mathrm{L}$ of cell media were then taken off and replaced with $100 \mu \mathrm{L}$ of solutions of the different compounds of interest at $10 \mu \mathrm{M}$ (final concentration in wells: $5 \mu \mathrm{M}$ ). Spheroids were left to incubate at $37^{\circ} \mathrm{C}$ with $5 \% \mathrm{CO}_{2}$ for 24 or $48 \mathrm{~h}$. LysoTracker Deep Red and Hoechst 33342 (nuclei stain) were added $1 \mathrm{~h}$ before imaging. Media were subsequently removed and the spheroids were washed with warm PBS three times. Spheroids were eventually transferred into a 10 well confocal microscopy plate with colorless media for imaging. Confocal microscopy images were taken on a Leica TCS SP5 (Carl Zeiss, Germany) at a temperature of $37^{\circ} \mathrm{C}$, using a $\times 20$ objective and sequential scanning for each channel. $z$ stacks were acquired over a length of $100 \mu \mathrm{m}$, with a step of $2 \mu \mathrm{m}$ (50 steps overall). Excitation/emission procedure used for imaging is as follows: nucleus channel (405/406-459 nm), RFP channel for Cy3 compounds (561/557-598 nm), and LysoTracker Deep Red channel $(633 / 646-698 \mathrm{~nm})$.

Colocalization was measured using ImageJ to obtain Pearson's coefficients on a 3D projection of different spheroids. Details of the processing of the images can be found in the Supporting Information.

\section{RESULTS AND DISCUSSION}

Design and Synthesis of the Library of Cyclic Peptide-Polymer Conjugates. In order to study the effect of different design parameters on the cellular uptake of CPNTs (Scheme 1), two cyclic peptides with different potential for polymer arm incorporation and three hydrophilic polymers with different composition and morphology were chosen. Cyclic peptides were synthesized by cyclizing and deprotecting linear peptides obtained from automated solid-phase synthesis, in a similar fashion to that previously reported in the literature (see Supporting Information for the experimental procedure) ${ }^{28}$ The amino acid sequences considered herein are similar to the ones used in previous studies on the selfassembly of CPNTs. ${ }^{20} \mathrm{CP} \mathbf{1}$ and $\mathbf{2}$ contained four D-leucine segments, essential for the self-assembly, in alternation with Llysine acting as the reactive arms (one or two depending on the peptide) and L-tryptophan ( 2 or 3 units, acting as a UV chromophore and further stabilizing the stacking process).

Several different systems were used to form the polymeric arms in this study. Linear poly(ethylene glycol) (PEG) was chosen as it is present in a high number of pharmaceutical preparations, given its biocompatibility and the large amount of information on its safety profile in vivo. ${ }^{29} \mathrm{~A}$ brush copolymer of PEG-acrylate 480 (PEGA) was also synthesized for comparison, to evaluate the impact of morphology on both self-assembly and cellular uptake. Investigating alternatives to PEG is of interest, given rising concerns on the immunogenicity of PEG. As such, poly-2-ethyl-2-oxazoline (PEtOx) was used as it is currently considered as a viable alternative to PEG given its biocompatibility, and allows easier access to diverse structures. ${ }^{30,31}$ Finally, all $\mathrm{CP}$-polymer conjugates were labeled using cyanine 3 , as conjugates with this dye have already shown good potential for biological imaging. ${ }^{32}$ Nonlabeled conjugates were synthesized for characterization of the self-assemblies by scattering techniques, given the prohibitive cost of fluorescent labels limiting the reaction scale. All the chromatograms mentioned from here are presented in Figure S1 in the Supporting Information.

Cationic ring opening polymerization (CROP) was performed in a microwave reactor using 2-ethyl-2 oxazoline as a monomer to form the PEtOx arms, adopting an end-group functionalization strategy with a tert-butyl-protected carboxylic acid on the initiator end (12) and a xanthate group on the terminating agent end. Bifunctional poly(2-ethyl-2-oxazoline) 13 was formed by CROP of 2-ethyl-2-oxazoline, with excellent control over the polymerization $\left(M_{\mathrm{n}}\right.$ nuclear magnetic resonance $(\mathrm{NMR})=5688 \mathrm{~g} / \mathrm{mol}, M_{\mathrm{n}}$ size exclusion chromatography $($ SEC $)=6150 \mathrm{~g} / \mathrm{mol}$, and $B=1.16$ ) and high end-group fidelity, as determined by mass spectrometry (MS) and NMR (see Supporting Information, Figures S5 and S6 for NMR, S10 for mass spectrometry). Moreover, end groups were chosen to allow the orthogonality of the reactions involved in the following modification. Both protecting groups were removed after treatment under basic conditions, allowing for further functionalization with a maleimide-functionalized cyanine 3 on the thiol-end to form polymer 3 . The polymer was attached on the cyclic peptides, to form conjugates 6 and 7.

A commercial $\mathrm{N}$-hydroxysuccinimide (NHS) and Bocprotected amine bifunctional $10 \mathrm{~kg} / \mathrm{mol}$ PEG was used to synthesize cyanine 3-functionalized CP-PEG systems; these groups were chosen because of their orthogonality. The polymer was first conjugated onto the cyclic peptide using activated amide coupling, in the presence of N,N-diisopropylethylamine as a hindered base. Yields for this reaction were $65 \%$ for both cyclic peptides involved in this study, and dialysis against pure water allowed for an excellent recovery of the conjugate while removing the free unreacted polymer. The conjugates were then treated with a cleavage mixture of TFA/ TIPS/water to deprotect the amine groups, prior to functionalization of the pendant amine groups using NHSmodified cyanine 3 . The conjugates were then purified to remove any free dye or fluorescent polymer using dialysis in water and preparative high-performance liquid chromatography (HPLC), affording conjugates 8 and 9. A control fluorescent PEG of $20 \mathrm{~kg} / \mathrm{mol}$ was also synthesized for biological studies using the same labelling technique, as described in the Supporting Information.

Fluorescently labeled poly(PEGA) systems were produced using reversible addition-fragmentation chain transfer (RAFT) polymerization. RAFT polymerization is a living radical polymerization process, allowing excellent control over the molecular weight and dispersity of the polymer synthesized. ${ }^{33}$ A statistical copolymer of PEGA and $N$-acryloxysuccinimide (NAS), a reactive monomer that can be used for postfunctionalization with amines, was first synthesized using (propanoic acid)yl butyl trithiocarbonate RAFT agent 18 given its compatibility with acrylates (the $\mathrm{R}$ group leading to the formation of a secondary radical). Brush copolymer 19 $\left[M_{\mathrm{n}}(\mathrm{SEC})=8,400 \mathrm{~g} / \mathrm{mol}, M_{\mathrm{n}}(\mathrm{NMR})=9916 \mathrm{~g} / \mathrm{mol}\right.$, and $D$ $=1.14]$, containing an average of 23 units of PEGA and 2.5 units of NAS as indicated by NMR, was obtained with excellent control over the polymerization. Amine-functionalized cyanine 3 was then used to label the copolymer and form polymer 5; HPLC with fluorescence detection and NMR analysis (peaks in the aromatic regions) confirmed successful labeling. Both polymeric compounds were conjugated to the cyclic peptides using activated amide coupling (using NHS 
a)

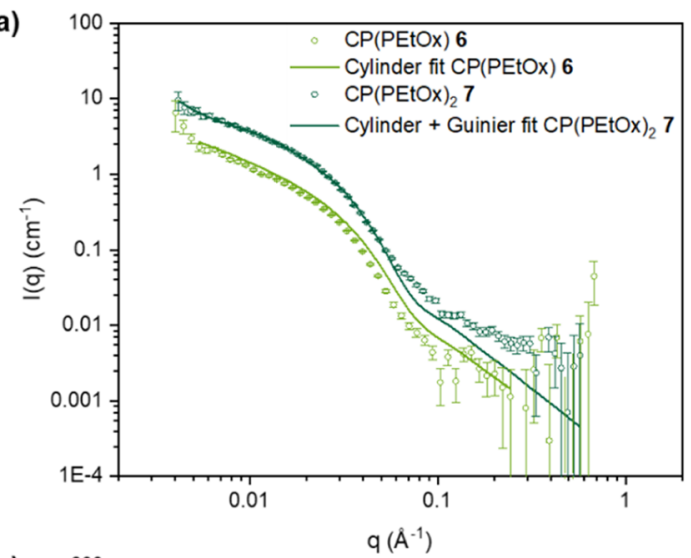

c)

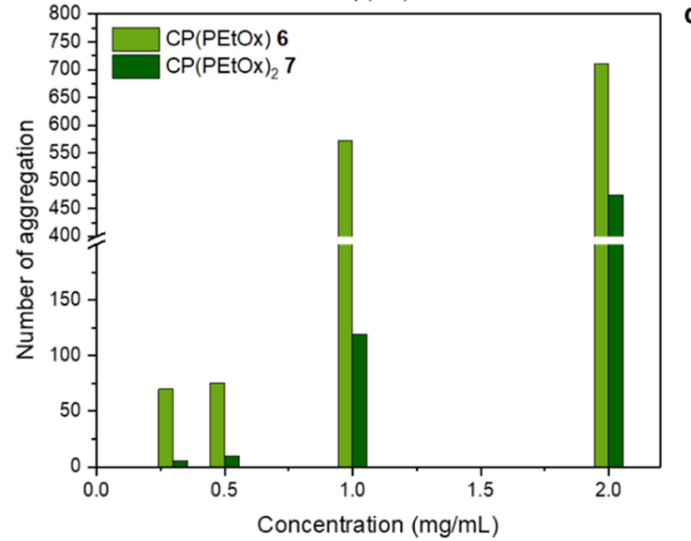

b)

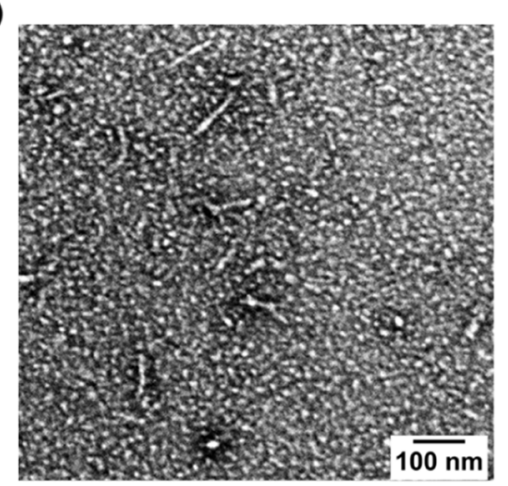

d)

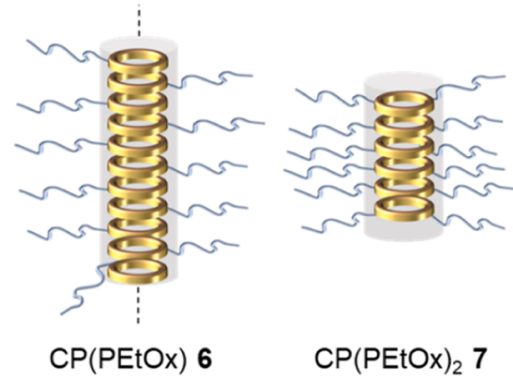

Figure 1. Self-assembly of PEtOx CP-polymer conjugates. (a) SANS cross section of the self-assembly of PEtOx conjugates 6 (light green) and 7 (dark green) at $2 \mathrm{mg} / \mathrm{mL}$ in $\mathrm{D}_{2} \mathrm{O}$. Hollow circles are used for the data plots, fits are plotted as lines and the error bars are statistical. (b) TEM image representing morphologies obtained from a $\mathrm{CP}(\mathrm{PEtOx})_{2} 7$ sample (stained with UOAc). (c) Evolution of the number of aggregation with concentration, determined by SLS for PEtOx conjugates $(6$ and $7,0.3-2.0 \mathrm{mg} / \mathrm{mL})$. Corresponding Zimm plots can be found in the Supporting Information in Figure S17. (d) Schematic representing the morphologies determined for PEtOx-CP polymer conjugates.

esters or O-(1H-6-Chlorobenzotriazole-1-yl)-1,1,3,3-tetramethyluronium hexafluorophosphate/4-Methylmorpholine (HCTU/NMM) as an activating agent) and then purified to remove any free dye or fluorescent polymer using dialysis in water and preparative HPLC. The HPLC chromatograms showed little difference in the retention time between the different conjugates, as all the compounds eluted around $80 \%$ $\mathrm{MeOH}$ content in the gradient tested, suggesting they were all of similar lipophilicity; this result is further corroborated by a detailed study by Grube et al., demonstrating the similarities between PEG and PEtox in terms of physicochemical properties. $^{34}$ It is hypothesized that extensive self-assembly of $\mathrm{CP}(\mathrm{PEtOx})$ explains the peculiar chromatograms for this compound (see in the Supporting Information, Figure S1 for the chromatograms and Table S1 for the SEC data summary).

Characterization of the Supramolecular Self-Assemblies. The size and shape of the self-assembled species formed by the previously synthesized $\mathrm{CP}$-polymer conjugates in $\mathrm{D}_{2} \mathrm{O}$ were evaluated using both SANS and static light scattering (SLS). SANS is a powerful technique to characterize supramolecular assemblies. It has been previously used on $\mathrm{CP}$-polymer based systems, to determine the size and morphologies of the assemblies. ${ }^{20}$ Here, SANS was used to establish how changes in the polymer corona on the CPNT affected the self-assembly process. An estimation of the size and morphology of $\mathrm{CP}$-polymer conjugates in aqueous solution can be obtained by fitting the data to appropriate models. Different models were tested in the fitting process; the best fit was selected using the Akaike information criterion (AIC) to account for the variation in the number of parameters in each model (for more details, please refer to the Supporting Information and Table S2 for the AIC calculations). The structures studied here showed the best fits to the SASFit cylindrical micelle (with Gaussian polymer chains) form factor CYL + Chains(RW), with the exception of CP(PEG) 9 and CP- $[\operatorname{poly}(\mathrm{PEGA})]$ 10, which had the best fit to a Benoit polymer star form factor. ${ }^{26,35} \mathrm{CP}-[\text { poly }(\mathrm{PEGA})]_{2} 11$ was not measured, as it has previously shown to give very little selfassembly. $^{20}$

For concentrations of $2 \mathrm{mg} / \mathrm{mL}$, PEtOx conjugates 6 and 7 formed long tubular assemblies, confirmed by the scattering profiles. Higher concentrations could not be measured because of the lack of solubility. At low $q$ values, there was no plateau reached, suggesting that the overall size of the tube was outside the window of observation for this SANS experiment (150 $\mathrm{nm}$ ) for $\mathrm{CP}(\mathrm{PEtOx}) 6$ (see Figure 1). An apparent length of $26.6 \mathrm{~nm}$ was found for $\mathrm{CP}(\mathrm{PEtOx})_{2}$, in accordance with the previous results demonstrating the effect of the number of polymer arms on a CP on self-assembly; data at lower $q$ are necessary to confirm this value given the lack of a clear plateau at the lowest $q$ values measured. ${ }^{20}$ An additional contribution, based on an extended Guinier form factor, was implemented in the fit for the low $q$ region. The upturn at low $q$ was thus incorporated in the model (to represent the potential presence of further aggregation), similar to what has been reported on some previously reported CPNTs described by Mansfield et 
a)

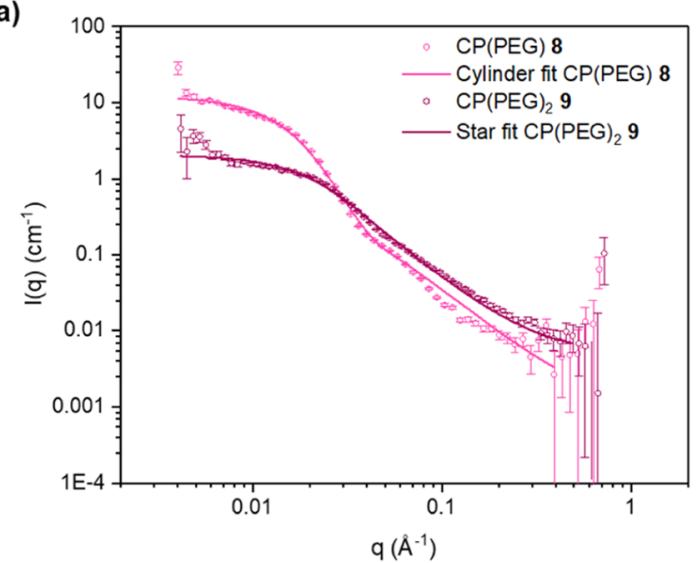

c)

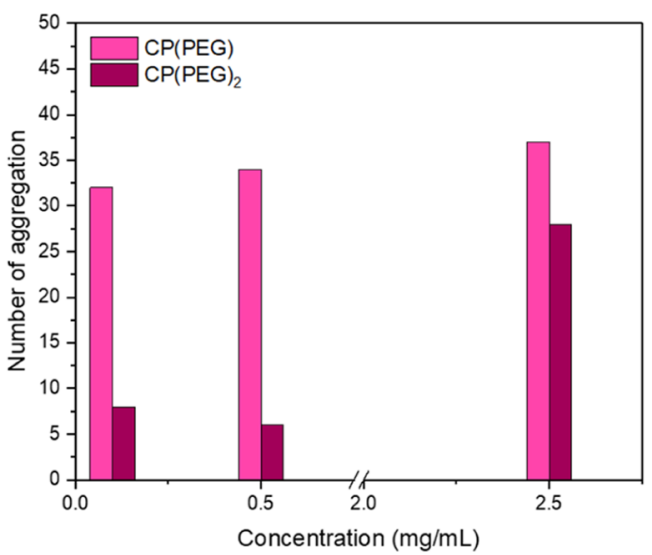

b)

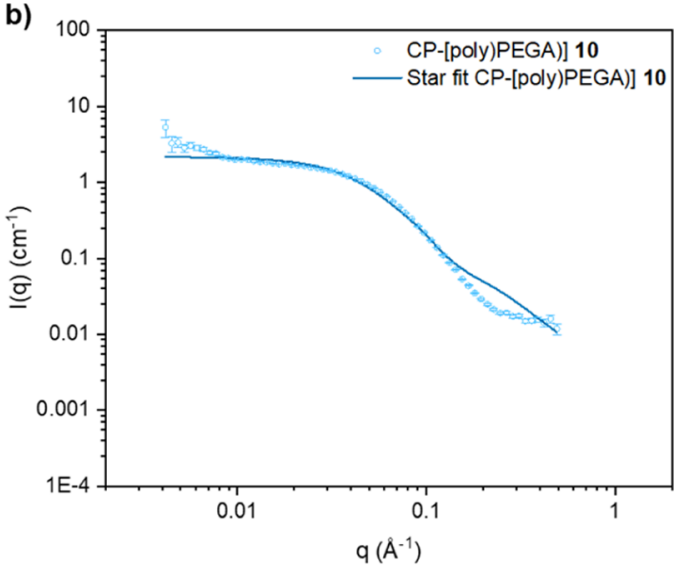

d)
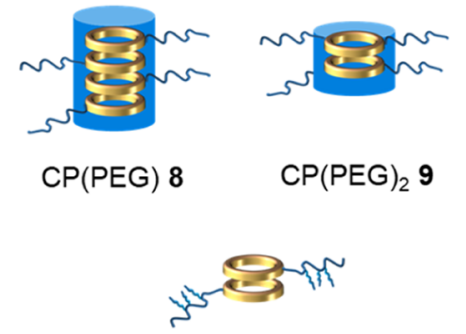

CP-poly(PEGA) 10

Figure 2. Self-assembly of PEG and poly(PEGA) conjugates. (a) SANS cross-section of the self-assembly of PEG conjugates 8 (pink) and 9 (purple) at $5 \mathrm{mg} / \mathrm{mL}$ in $\mathrm{D}_{2} \mathrm{O}$. (b) SANS cross-section of the self-assembly of CP-[poly(PEGA]) 10 (blue) at $5 \mathrm{mg} / \mathrm{mL}$ in $\mathrm{D}_{2} \mathrm{O}$. Hollow circles are used for the data plots, fits are plotted as lines, and error bars are statistical. (c) Evolution of the number of aggregation with concentration, determined by SLS for PEG conjugates $(8$ and $\mathbf{9}, 0.1-2.5 \mathrm{mg} / \mathrm{mL}$ ). (d) Schematic representing the morphologies determined for PEG and poly(PEGA) CP-polymer conjugates.

al. ${ }^{20}$ SLS was used to probe the SANS data further, as it allows access to complimentary low $q$-values; the data were used to estimate of the length of the self-assemblies at the working concentration used for cellular uptake experiments. SLS of $\mathrm{CP}(\mathrm{PEtOx})$ confirmed lengths between 35 and $435 \mathrm{~nm}$, in a range of concentrations varying between 0.3 and $2 \mathrm{mg} / \mathrm{mL}$, which would explain why an accurate length could not be obtained from SANS at $2 \mathrm{mg} / \mathrm{mL}$. The concentration dependence observed by SLS could be explained by thermodynamic considerations. The strong self-assembly observed could be linked to the hydrogen bonding between the polymer chains and water, thus affecting the self-assembly process. First, the polymer chain length is relatively low, reducing the adverse contribution of steric hindrance to selfassembly. Second, compared to other PEG based conjugates, the PEtOx conjugates are most likely less solvated in water as it would be expected from the study carried out by Grube et al. comparing PEtOx and PEG polymers of similar molecular weight. $^{34}$ This lower solvation may, therefore, boost the selfassembly process as water is less likely to be solvating the polymer corona. As a result, the $\mathrm{CP}-\mathrm{CP}$ interaction is less hindered and $\mathrm{CP}(\mathrm{PEtOx})$ unimers may be able to compensate for the loss of conformational entropy because of chain stretching in the self-assembly process, following models previously described by Wang and Safran on polymers with head groups prone to self-assembly. ${ }^{36}$ Further growth is, therefore, possible at higher concentrations (with respect to solubility in water). Those results need, however, to be considered with caution, as there is a higher uncertainty on the Zimm plots at low concentrations because of low scattering intensities. Another SANS measurement performed on CP(PEtOx) at $0.2 \mathrm{mg} / \mathrm{mL}$ showed a similar profile to that for higher concentrations, with nanotubes of high length that could not be accurately determined (see Supporting Information, Figure S15).

Furthermore, transmission electron microscopy (TEM) of $\mathrm{CP}(\mathrm{PEtOx})_{2}$ showed the presence of some tubular structures with a length of 75-80 $\mathrm{nm}$. Other morphologies were also observed by TEM imaging; their exact nature could, however, not be identified given the poor contrast obtained with these $\mathrm{CP}$-polymer conjugate species (see summary of the data in Figure 1).

As for linear PEGylated CPs, the SANS data (for a $5 \mathrm{mg} / \mathrm{mL}$ concentration) suggested the presence of tubular assemblies with a length of $15.8 \mathrm{~nm}$ [CP(PEG) 8] (see Figure 2). The choice of the cylindrical micelle fitting model was supported by AIC calculations and by previous characterization work on PEG CPNTs where the PEG chains of different molecular weights ( 2 and $20 \mathrm{~kg} / \mathrm{mol}$ ) were used. ${ }^{20,32}$ The best fit for $\mathrm{CP}(\mathrm{PEG})_{2} 9$ was obtained for a very short cylindrical micelle (0.8 nm long) following the AIC calculations; however, given the very low aspect ratio $(<1)$ determined, it was decided to 
Table 1. Summary of the Self-Assembly Data Collected Using Scattering Techniques (SANS and SLS) ${ }^{a}$

\begin{tabular}{|c|c|c|c|c|c|c|}
\hline compound & fit & $\begin{array}{c}\text { length (SANS, } \\
\mathrm{nm})\end{array}$ & $\begin{array}{l}\text { concentration } \\
(\mathrm{mg} / \mathrm{mL})\end{array}$ & $\begin{array}{c}N_{\text {agg }} \text { SLS at } 0.1-0.3 \\
\mathrm{mg} / \mathrm{mL}\end{array}$ & $\begin{array}{l}\text { corresponding lengths } \\
(\mathrm{nm})\end{array}$ & $\begin{array}{l}\text { aspect ratio (calculated from } \\
\text { SANS data) }\end{array}$ \\
\hline $\mathrm{CP}(\mathrm{PEtOx}) 6$ & $\mathrm{CM}$ & $>150$ & 2 & 75 & 35.0 & 25.3 (length from SLS) \\
\hline $\mathrm{CP}(\mathrm{PEtOx})_{2} 7$ & $\mathrm{CM}$ & 26.6 & 2 & 5 & 2.35 & 7.6 \\
\hline $\mathrm{CP}(\mathrm{PEG}) 8$ & $\mathrm{CM}$ & 15.8 & 5 & 32 & 15.0 & 2.3 \\
\hline $\mathrm{CP}(\mathrm{PEG})_{2} 9$ & SP & $14^{*}$ & 5 & 8 & 3.76 & \\
\hline CP-[poly(PEGA)] 10 & SP & $8.3^{*}$ & 5 & & & \\
\hline $\mathrm{CP}-[\operatorname{poly}(\mathrm{PEGA})]_{2} \mathbf{1 1}^{20}$ & SP & $2^{*}$ & 5 & & & \\
\hline
\end{tabular}

favor the star polymer fit in this case (a cylindrical fit would not be the most sensible for such values of aspect ratio).

SLS was used again to determine the length of PEG CPNTs; the data were found to be quite consistent with the values from SANS for $\mathrm{CP}(\mathrm{PEG})$ and $\mathrm{CP}(\mathrm{PEG})_{2}$ self-assemblies at high concentration; $\mathrm{CP}(\mathrm{PEG})_{2}$ appeared to form nanotubes with a length slightly dependent on the concentration (varying from $3.7 \mathrm{~nm}$ at low concentration to $13 \mathrm{~nm}$ at $2.5 \mathrm{mg} / \mathrm{mL}$ ). Those changes in aggregation with concentration were found to be relatively minor for $\mathrm{CP}(\mathrm{PEG})$ in comparison with $\mathrm{PEtOx}$ conjugates, which could be explained by the polymer crystalline behavior potentially stabilizing the self-assembly and by the higher DP of PEG (225), inducing an increased steric hindrance in the system, therefore, limiting the compensation of any loss of conformational entropy.

$\mathrm{CP}$-polymer conjugates with a brush polymer poly(PEGA) had a different behavior to the their linear counterparts. The SANS profile of CP-[poly(PEGA)] 10 was best fitted to a star polymer model, according to the AIC calculations (see Figure 2 ). A length per se could not be determined with the star polymer model; however, $N_{\text {agg }}$ was calculated from the scale factor and found to be around 8 (see more details in the Supporting Information and Table S8). Unfortunately, accurate Zimm plots of poly(PEGA) conjugates could not be obtained by SLS given the very low levels of self-assembly. The data for CP- $[\text { poly }(\mathrm{PEGA})]_{2}$ were directly taken from the work of Mansfield et al. who previously showed the lack of selfassembly for this system. ${ }^{20}$ The aspect ratio of each compound was calculated following the procedure detailed in the Supporting Information (Figure S16), with values varying from $2.3[\mathrm{CP}(\mathrm{PEG})]$ to $25.3[\mathrm{CP}(\mathrm{PEtOx})]$. All data related to self-assembly are summarized in Table 1.

Cellular Uptake. The cellular uptake of the aforementioned labeled $\mathrm{CP}$-polymer conjugates was evaluated on two different cell lines, breast cancer cell line MDA-MB-231, and murine fibroblasts NIH 3T3. The relative fluorescence within cells treated with each of the compounds was measured using the pictures acquired by fluorescence microscopy on a plate reader. Images were corrected according to the intrinsic fluorescence of each of the compounds (details of image processing are presented in the Supporting Information). The fluorescence per field of view was evaluated under four sets of conditions, varying the concentration as well as the incubation time, allowing for a relative comparison between the CPNTs and polymers (Figure 3). As expected, both cell lines showed a positive uptake of the compounds, with a longer incubation time $(24 v s 2 \mathrm{~h})$ and a higher concentration $(25 v s 5 \mu \mathrm{M})$ leading to higher cellular uptake in both cell lines.

Different trends can be extracted from the cellular uptake study of this library of $\mathrm{CP}$-polymer conjugates. Variations in the intracellular fluorescence matched the apparent differences in self-assembly behavior, the extent of the self-assembly seemingly driving the rate of cellular uptake. The compounds have been regrouped into subcategories, depending on the nature of the polymer considered (PEtOx, linear PEG, or poly(PEGA)). Starting with $\mathrm{PEtOx} \mathrm{CP}$-polymer conjugates, it has been noticed that there is a significant preference in uptake for $\mathrm{CP}(\mathrm{PEtOx})_{2}$, compared to $\mathrm{CP}(\mathrm{PEtOx})$ and the free polymer chain in both cell lines, for the highest concentration and incubation time. The mean fluorescence recorded was 1.4 and 1.8 times higher for $\mathrm{CP}(\mathrm{PEtOx})_{2}$ compared to PEtOx and $\mathrm{CP}(\mathrm{PEtOx})$, respectively, in $\mathrm{NIH}$ 3T3. The same differences can be extracted from the data on breast cancer cells MDA$\mathrm{MB}-231$, with an increase by a factor of $1.9[\mathrm{CP}(\mathrm{PEtOx})]$ or 2.7 (PEtOx). We hypothesize that the size of the $\mathrm{CP}(\mathrm{PEtOx})$ nanotubes is most likely too high to be taken up efficiently in the timeframe considered, the SANS data indicate the presence of long nanotubes for $\mathrm{CP}(\mathrm{PEtOx}) 6$ even at $0.3 \mathrm{mg} / \mathrm{mL}$. This phenomenon becomes more obvious when increasing the concentration of material, matching the concentration-dependent self-assembly of $\mathrm{CP}(\mathrm{PEtOx})$, similar to previously investigated systems. ${ }^{32} \mathrm{CP}(\mathrm{PEtOx})_{2}$, forming seemingly smaller nanotubes than $\mathrm{CP}(\mathrm{PEtOx})$, is taken up more readily at higher concentrations. This is in accordance with previous observations that cylindrical structures of a very high aspect ratio are taken up at a slower pace than lower aspect ratio nanotubes, as membrane-wrapping is more energetically challenging for longer nanotubes. ${ }^{13}$ Alternative hypotheses were contemplated in the interpretation of those cellular uptake results, given the complexity to determine the morphologies and size of the self-assemblies at low concentrations with accuracy (as well as the potential presence of free unimers). A preferential interaction between CP(PEtOx) and lipid bilayers, because of the presence of cyclic peptides, could explain the results being obtained. Some cyclic peptides and CPNTs with Trp residues have previously shown potential for membrane interaction within bilayers, which in this case could be further accentuated given the slight lipophilicity of PEtOx. ${ }^{31}$

Such an interaction may not be present for polymer coronas that are more solvated; Danial et al. have shown that CPNTs with hydrophilic polymer coronas rarely induce any membrane interaction on large unilamellar vesicle (LUV) Assays. ${ }^{37}$

In the case of linear PEG conjugates, there was a significantly higher uptake in both cell lines studies of $\mathrm{CP}(\mathrm{PEG})$ ( $\sim 16 \mathrm{~nm}$ long) than for smaller assemblies formed by its two-arm counterpart $\mathrm{CP}(\mathrm{PEG})_{2}$, or a labeled $\mathrm{PEG}$ chain of $20,000 \mathrm{~g} / \mathrm{mol}$ [equivalent in molecular weight to $\mathrm{CP}$ $\left.(\mathrm{PEG})_{2}\right]$. The uptake of $\mathrm{CP}(\mathrm{PEG})$ is between three $(24 \mathrm{~h}, 25$ $\mu \mathrm{M})$ and five $(2 \mathrm{~h}, 5 \mu \mathrm{M})$ times higher than that of $\mathrm{CP}(\mathrm{PEG})_{2}$ in MDA-MB 231, and three times higher on average for $\mathrm{NIH}$ 3T3. Similarly, $\mathrm{CP}(\mathrm{PEG})_{2}$ had a significantly higher uptake 
a)
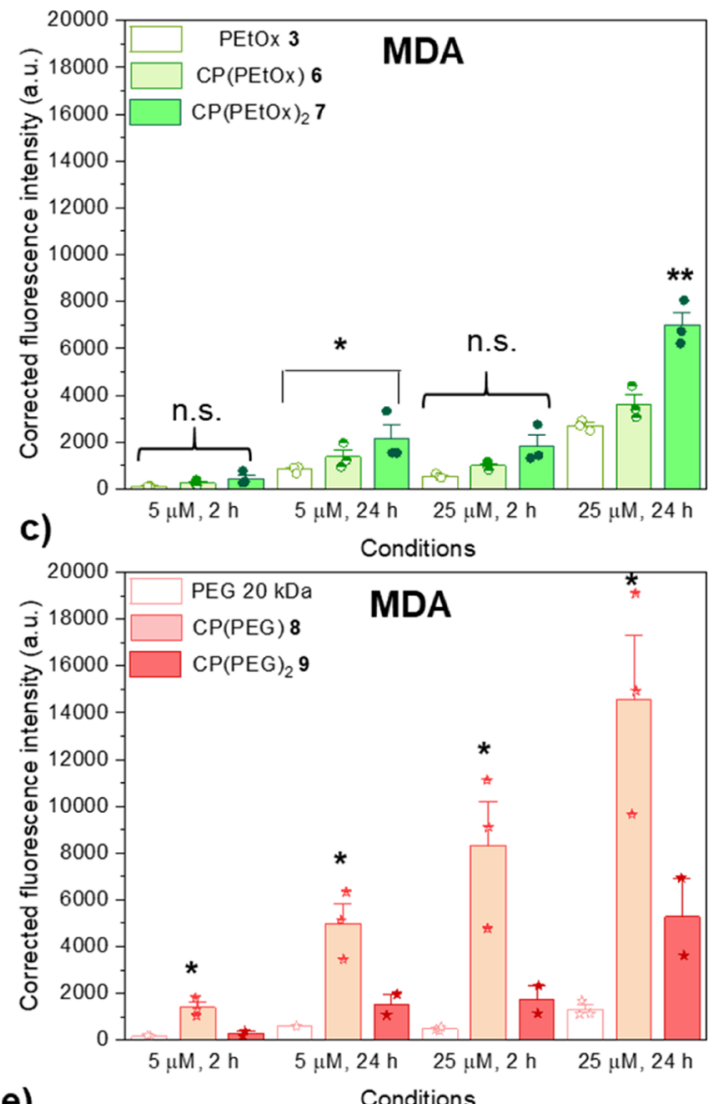

e)

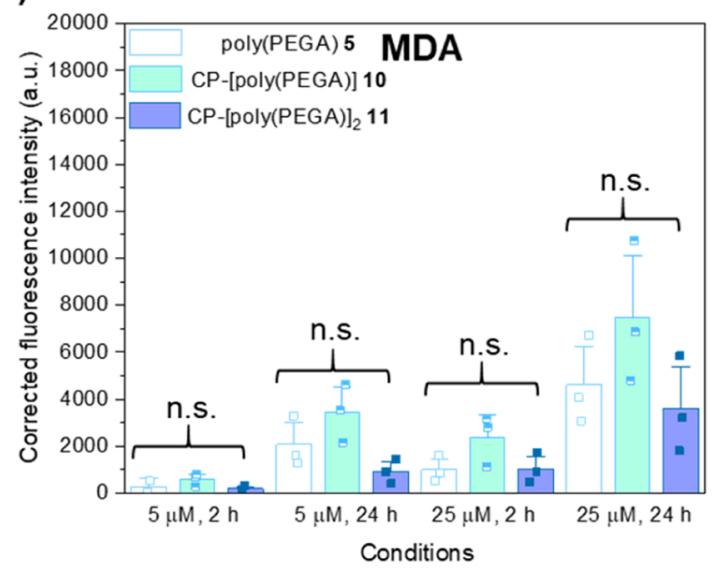

b)
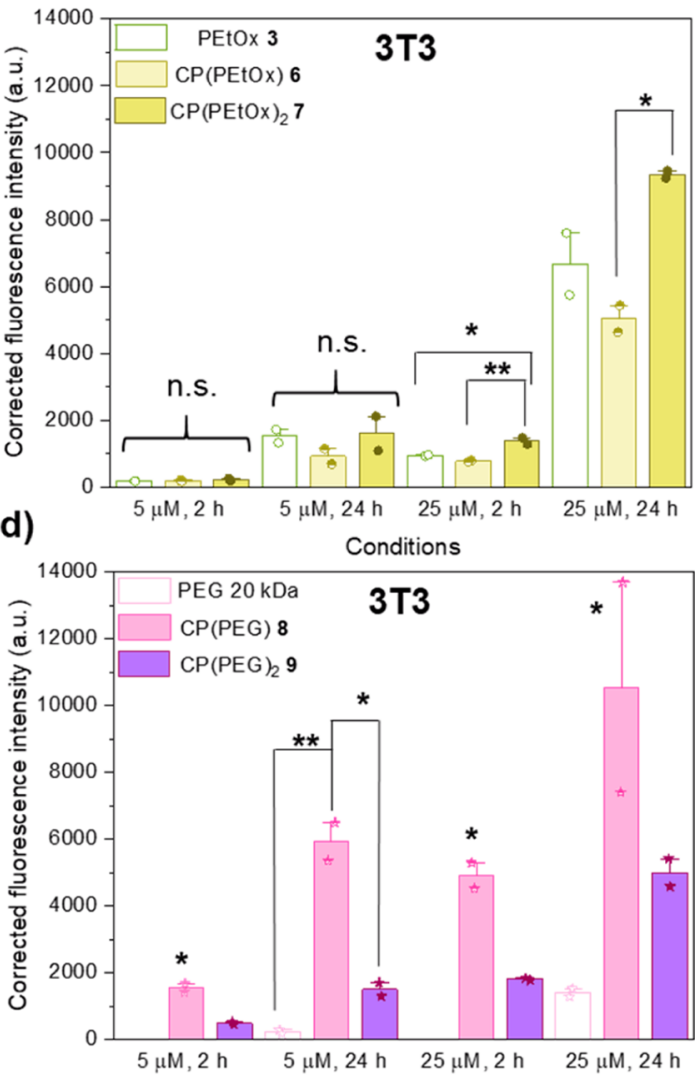

f)

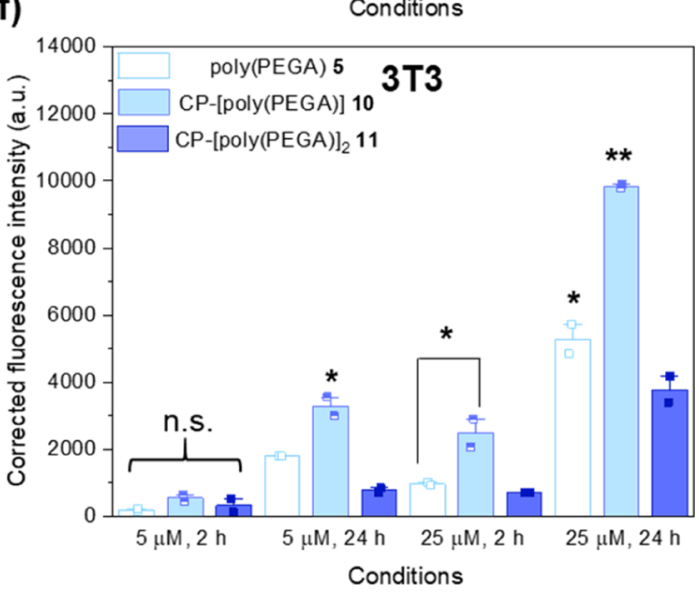

Figure 3. Evaluation of the cellular uptake of a library of $\mathrm{CP}$-polymer conjugates. (left) Cellular uptake in triple negative breast cancer cell line MDA-MB 231 following 2 or $24 \mathrm{~h}$ incubation in the presence of; 5 or $25 \mu \mathrm{M}$ of: (a) PEtOx polymer and CP(PEtOx) conjugates (3, 6, and 7); (c) $\mathrm{CP}(\mathrm{PEG})$ conjugates (8 and $\mathbf{9})$ and PEG $20 \mathrm{kDa}$ control; and (e) poly(PEGA) and CP-[poly(PEGA)] conjugates (5, 10, and 11). (Right) Cellular uptake in murine fibroblasts NIH 3T3 (same compounds and conditions). The data were plotted as bar charts (mean) with an overlap of all repeats. Error bars represent the standard error of the mean $(n=3$ or 2). Discrepancies in intrinsic fluorescence were corrected using fluorescence coefficient for each individual compound in PBS buffer. (see Supporting Information, Table S10). Statistical significance was assessed by an ANOVA + Tukey-Kramer posthoc test. *: $p<0.05, * *: p<0.005$.

than its linear polymer counterpart, suggesting again a beneficial impact of the self-assembly induced by the cyclic peptide core. Furthermore, those variations in uptake between the different PEG species, which appeared more pronounced than for PEtOx and PEGA conjugates with 1 or 2 polymer arms, were attributed to a difference in their propensity to selfassemble. It was also linked to the presence of a bulky second polymer arm, which makes it more challenging for the system to be taken up.
As for brush poly(PEGA) conjugates, no significant differences in uptake in MDA-MB-231 were noticed. Such variations could be observed in NIH 3T3 (with the exception of low concentration and short incubation time), with a better entry of CP-[poly(PEGA)] compared to the free polymer and CP-[poly(PEGA) $]_{2}$. This increase in uptake is of a factor varying between $2.6(24 \mathrm{~h}, 25 \mu \mathrm{M})$ and $4.5(2 \mathrm{~h}, 25 \mu \mathrm{M})$. Notably, poly(PEGA) was taken up more readily than CP$[\text { poly }(\mathrm{PEGA})]_{2}$. Given that CP-[poly(PEGA) $]_{2}$ does not assemble and seems to behave like a long unimeric polymer 
a)
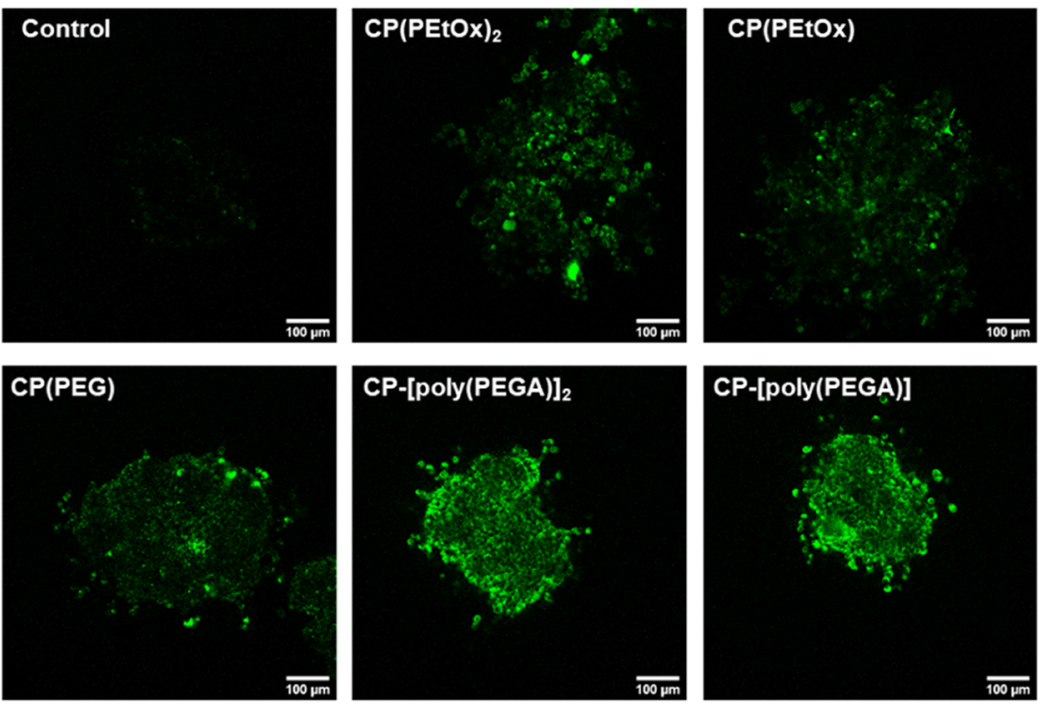

b)

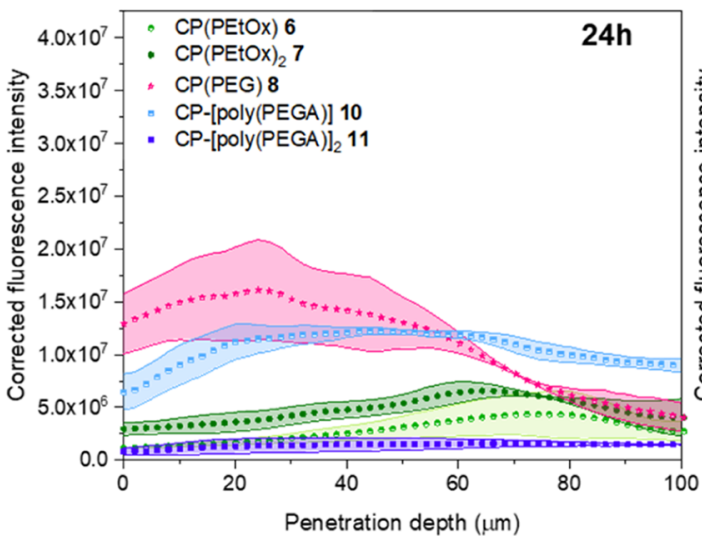

c)

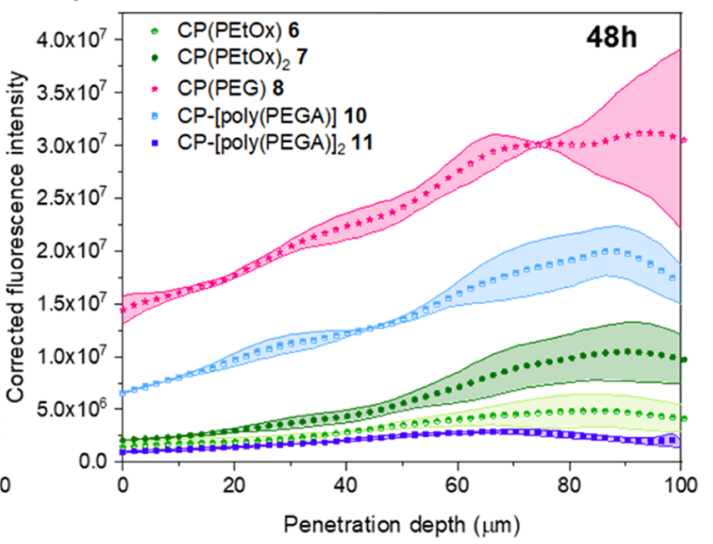

Figure 4. Evaluation of the potential for penetration in MCTS for different cyclic peptide-polymer conjugates. (a) CLSM penetration of CPpolymer conjugates $(11,10,8,7$, and 6) at $z=50 \mu \mathrm{m}$ in MCTS after $24 \mathrm{~h}$ incubation at $5 \mu \mathrm{M}$, Cy3 channel (green). The images were not corrected by the intrinsic fluorescence of each compound in buffer. (b) Penetration profile at $24 \mathrm{~h}$ and (c) $48 \mathrm{~h}$ for a series of CP-polymer conjugates $(6,7,8,10$, and 11$)$ over a section of $100 \mu \mathrm{m}$ of the spheroid. Each point represents the average relative fluorescence intensity recorded at a given $z$-stack in the spheroid, delimited by a region of interest. The envelope around each plot represents the standard error of the mean associated with each point (two replicates per timepoint, $n=2$ ).

chain $(\sim 20 \mathrm{kDa})$, it does not benefit from the boost in cellular uptake previously observed for $\mathrm{CP}(\mathrm{PEG})_{2}$ and $\mathrm{CP}(\mathrm{PEtOx})_{2}$. Again, the steric hindrance caused by the presence of a second polymer chain could explain the results obtained here and why the poly(PEGA) polymer is outperforming CP-[poly(PEGA) $]_{2}$ in terms of uptake in $3 \mathrm{~T} 3$ cells. Those results could also reinforce the hypothesis that self-assembly may be the driving force behind the preferential uptake of $\mathrm{CP}(\mathrm{PEtOx})_{2}$ at $25 \mu \mathrm{M}$, as steric hindrance did not seem to adversely affect it. Overall, self-assembly had a noticeable influence on the cellular uptake of CPNTs, with slight variations between the cell lines. CPNTs of a moderate length $(10-30 \mathrm{~nm})$ have the best uptake, compared to conjugates that self-assemble too readily [CP$(\mathrm{PEtOx})]$ or that do not form nanotubes (CP-[poly$($ PEGA $\left.)]_{2}\right)$. Steric hindrance is also to be accounted for to explain some of the patterns observed in particular for lowassembling poly(PEGA) conjugates (Figure 3). Cellular uptake was also assessed at low temperature to understand the contribution of energy-dependent mechanisms to the uptake of compounds presented in this work. Overall, our study on prostate cancer PC-3 cells showed that there is less uptake at 4 ${ }^{\circ} \mathrm{C}$ than at $37{ }^{\circ} \mathrm{C}$ as it was expected (as energy-dependent pathways are being knocked out). Cellular viability was assessed for all these systems using the XTT/PMS assay, evaluating changes in mitochondrial metabolism. None of the compounds previously tested was found to be toxic after $24 \mathrm{~h}$ incubation with concentrations up to $100 \mu \mathrm{M}$, further highlighting the biocompatibility of CPNTs (see in the Supporting Information, Figure S18).

Study of the Intracellular and Intraspheroidal Behavior by CLSM. Following on from our uptake and viability studies, the intracellular behavior of the different $C P$ conjugates was assessed using CLSM in both classic planar models (PC-3 cells) and 3D models [PC-3 multicellular tumor spheroids (MCTS)]. In both cases, localization studies were performed to determine where the conjugates could be found within a cell following $24 \mathrm{~h}$. Upon visual inspection of pictures from the $2 \mathrm{D}$ models, it is clear that the conjugates (green channel) entered the cells and accumulated in the lysosomes (magenta channel). This was confirmed by mathematical 
a)
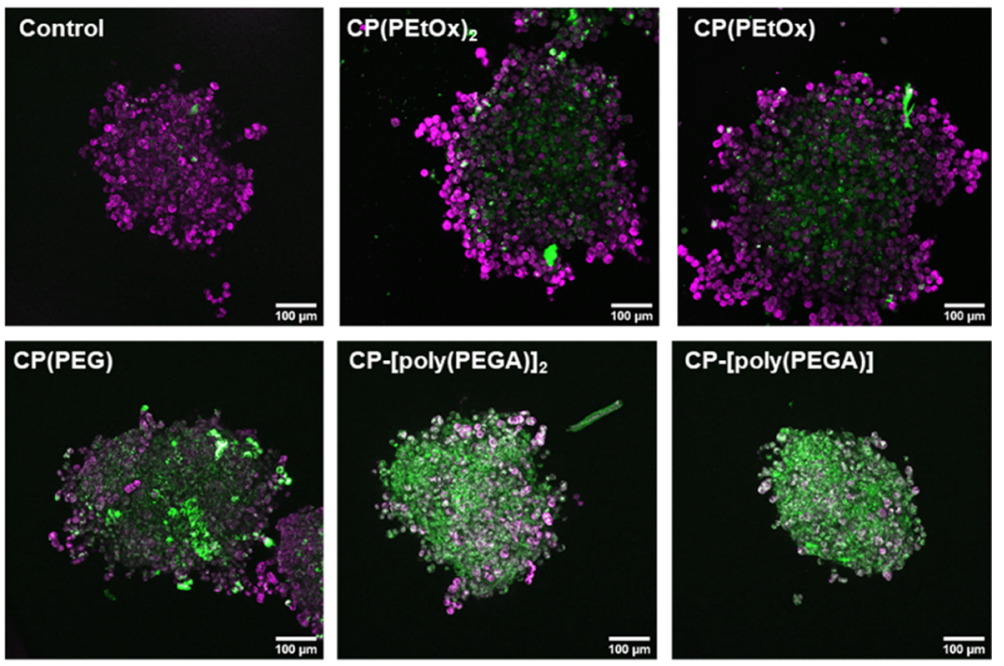

b)

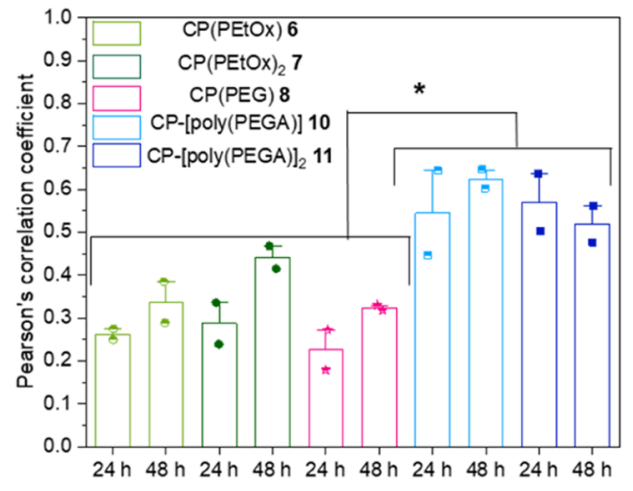

Figure 5. Evaluation of the lysosomal colocalization of cyclic peptide-polymer conjugates in MCTS. (a) Brightest point projection green/magenta overlay from $50 z$-stacks ( $2 \mu \mathrm{m}$ spacing) of PC-3 MCTS after $24 \mathrm{~h}$ incubation with a series of CP-polymer conjugates $(\mathbf{1 1}, \mathbf{1 0}, \mathbf{8}, 7$, and 6). Green represents the Cy3 channel of the conjugates, magenta is LysoTracker Deep Red, and gray-white areas indicate colocalization. (b) Evaluation of the overall level of colocalization between the green (Cy3 CP-polymer conjugate) and magenta (LysoTracker Deep Red) channels, using an average of the PCC between two independent spheroids. Bar-charts represent the mean with an overlap of all repeats. Error bars are the standard error of the mean on two replicates $(n=2)$. Statistical significance was assessed by an ANOVA + Tukey-Kramer post-hoc test. *: $p<0.05$.

analysis evaluating the correlation between the magenta (lysosomes) and green (CPNT) channels using the Pearson's correlation coefficients (PCCs). PCCs were measured using Just Another Colocalization Plugin (JACoP) ${ }^{38}$ on Fiji (ImageJ distribution $)^{39}$ for the channels of interest to quantify the colocalization of Cy3-labeled compounds with lysosomes in cells. The levels of lysosomal localization of the different conjugates varied from 0.27 ( $\mathrm{CP}(\mathrm{PEG}) 8$ ) to 0.59 (CP$\left.[\text { poly }(\mathrm{PEGA})]_{2} 11\right)$, suggesting different intracellular behavior depending on the self-assembly (see Supporting Information, Figure S19).

This phenomenon and the potential of the CPNT to achieve intratumoral penetration were extensively studied on PC-3 spheroids. MCTS are a more accurate model of tumor behavior than $2 \mathrm{D}$ models as they can be used to replicate different parameters such as the existence of intratumor gradients (oxygen, nutrients, $\mathrm{pH}$, etc.,) and cell-cell interactions. ${ }^{40,41}$ Penetration profiles for each compounds, based on the fluorescence intensity of each $z$-stack acquired, were plotted to compare the incubation times at 24 and $48 \mathrm{~h}$. Efficient penetration was observed at $24 \mathrm{~h}$, looking at the inside area of the spheroid ( $50 \mu \mathrm{m}$ in depth from the surface) for $\mathrm{CP}$-polymer conjugates. The visual patterns observed are different between cylindrical micelle-like CPNTs 6, 7, and 8 and star-like poly(PEGA) conjugates $\mathbf{1 0}$ and 11. In terms of relative fluorescence intensity (using the coefficients previously described in the uptake study in Table S10 of the Supporting Information), the trends are similar to what has been observed in the fluorescence microscopy study previously described (vide supra). CP(PEG) showed the highest fluorescence intensity overall in the spheroid and a two-fold increase in the internalized quantity between 24 and $48 \mathrm{~h}$. The fluorescence maximum was reached at a higher penetration depth in spheroids treated with the $\mathrm{CP}$-polymer conjugates for $48 \mathrm{~h}$, compared with those treated for $24 \mathrm{~h}$. This suggests that the diffusion through the core of the spheroid is timedependent as observed for cylindrical viral NPs by Steinmetz and co-workers (see Figure 4$).^{42}$

The intraspheroidal behavior also differed, with varying levels of colocalization between Lysotracker and the different $\mathrm{CP}$-polymer conjugates. Nanotube-forming conjugates [CP(PEtOx) 6, $\mathrm{CP}(\mathrm{PEtOx})_{2} 7$ and $\mathrm{CP}(\mathrm{PEG})$ 8] did not exhibit much lysosomal colocalization after $24 \mathrm{~h}$, according to the values of the PCC recorded, by comparison with compounds with low self-assembly [poly(PEGA) conjugates]. This can also be observed on the maximum projection for both channels (Cy3: green; LysoTracker: magenta) where more white areas can be observed in the case of poly(PEGA) conjugates. These 
data help to understand the mode of internalization used by CPNTs.

Transcellular propagation would involve the conjugates to be internalized and excreted by cells on the spheroids. It would, therefore, lead to higher lysosomal correlation than paracellular propagation, which should happen throughout the intracellular matrix of the tumor model. Overall, PCC values are on average two times lower for conjugates containing PEG and PEtOx than for poly(PEGA) conjugates. In terms of kinetics of the uptake phenomenon, it is interesting to notice that Pearson's coefficients for PEtOx and PEG conjugates are slightly higher after $48 \mathrm{~h}$ incubation, which supports the hypothesis of a delayed uptake via endocytosis for systems with higher aspect ratio. Such results seem to indicate that the transcellular uptake is the preferred mechanism for star-like poly(PEGA) conjugates. As for higher aspect ratio nanotubes (PEG and PEtOx based), the contribution of paracellular uptake is more important, as the lysosomal correlation coefficients are lower. Lower levels of colocalization for the $\mathrm{CP}(\mathrm{PEtOx})$ and $\mathrm{CP}(\mathrm{PEG})$ nanotubes tested can be explained by an accumulation of nanotubes in the cytosol, the intercellular spacing, or in other vesicular compartments of the cell. Accumulation in nonlysosomal areas of the spheroid could also be because of the presence of anoxic regions that might be more readily accessible and allow for higher uptake, following the known enhanced permeation and retention effect (see Figure 5). However, punctuation present on 2D models for all labeled compounds suggests a compartmentalization rather than an endosomal escape in the cytosol (see in the Supporting Information, Figure S19). The significant increase in colocalization over time for PEG and PEtOx nanotubes could be explained by a delayed transcellular mechanism. Care must be taken not to extrapolate those trends on uptake and penetration in model MCTS to what could happen in a real tumor. Despite their ability to provide substantial information on the behavior of CPNTs in a 3D cellular system, MCTS are limited when it comes to replicating cell-to-cell interaction as they exist in real tumors; they cannot perfectly mimic relatively tight intercellular junctions that can be encountered on tumors found in vivo, as it has been highlighted in a review by $\mathrm{Lu}$ and Stenzel. ${ }^{43}$

Overall, it is clear that the nature of the $\mathrm{CP}$-polymer conjugates influences the behavior in MCTS. In terms of overall fluorescence intensity, similarities can be found with the study on the planar models with a predominance of $\mathrm{CP}(\mathrm{PEG})$ and CP-[poly(PEGA)]. Colocalization studies showed a higher predisposition of nonassembling structures to accumulate within lysosomal compartments, while self-assembling conjugates were present in different areas (such as intercellular regions, see Figures 4 and 5).

\section{CONCLUSIONS}

In summary, a library of different fluorescently labeled CPNTs with one or two hydrophilic polymer chains was synthesized. The impact over a range of design parameters (number of polymer arms, nature of the polymer, and morphology) was studied, confirming their strong influence on the self-assembly process in water, allowing access to diverse nanostructures. The formation of self-assemblies of different sizes and shapes, varying from a couple of nanometer star-like structures (CP$\left.[\text { poly }(\mathrm{PEGA})]_{2}\right)$ to nanotubes with lengths over $100 \mathrm{~nm}$ $[\mathrm{CP}(\mathrm{PEtOx})]$, was linked to the variations in cellular uptake in mammalian cells. Overall, there is an optimal size range for efficient uptake of CPNTs to be aimed for, around 10-20 nm, as seen with $\mathrm{CP}(\mathrm{PEG})$ by comparison with shorter [poly(PEGA) based] or longer (PEtOx conjugates) self-assemblies; steric considerations have also been contemplated, suggesting a balance between steric hindrance and extent of self-assembly in the uptake phenomenon. Further investigations on the intratumoral behavior of the conjugates using MCTS models showed clear differences between the uptake of assembled and nonassembled structures. The maximal fluorescence intensity recorded increased quite significantly with time for $\mathrm{CP}(\mathrm{PEG})$, $\mathrm{CP}(\mathrm{PEtOx})_{2}$, and $\mathrm{CP}-[$ poly$(\mathrm{PEGA})]$, corroborating with the results from $2 \mathrm{D}$ model studies, showing a very efficient uptake of $\mathrm{CP}(\mathrm{PEG})$. As for lysosomal colocalization studies, CPNTs formed by poly(PEGA) conjugates showed higher colocalization in lysosomes than PEG and PEtOx nanotubes, suggesting differences in the uptake mechanism with different contributions of paracellular and transcellular uptake. This excellent penetration of CPNTs and the characteristics of this uptake phenomenon in $3 \mathrm{D}$ models are encouraging, suggesting that these systems have some potential for the delivery of small molecules in a tumor and should be further investigated in vivo.

\section{ASSOCIATED CONTENT}

\section{Supporting Information}

The Supporting Information is available free of charge at https://pubs.acs.org/doi/10.1021/acs.biomac.0c01512.

Synthetic procedures, characterization (chromatography, NMR, and mass spectrometry), details of the scattering data fitting process and fitting parameter values, additional cellular uptake experiments and viability assays, and details of the image processing (PDF)

\section{AUTHOR INFORMATION}

\section{Corresponding Author}

Sébastien Perrier - Department of Chemistry and Warwick Medical School, University of Warwick, Coventry CV4 7AL, U.K.; Faculty of Pharmacy and Pharmaceutical Sciences, Monash University, Parkville, VIC 3052, Australia; ○ orcid.org/0000-0001-5055-9046; Email: s.perrier@ warwick.ac.uk

\section{Authors}

Sean H. Ellacott - Department of Chemistry, University of Warwick, Coventry CV4 7AL, U.K.

Carlos Sanchez-Cano - Department of Chemistry, University of Warwick, Coventry CV4 7AL, U.K.; Center for Cooperative Research in Biomaterials (CIC biomaGUNE), Basque Research and Technology Alliance (BRTA), Donostia San Sebastián 20014, Spain; 이이.org/0000-0002-95220019

Edward D.H. Mansfield - Department of Chemistry, University of Warwick, Coventry CV4 7AL, U.K.

Julia Y. Rho - Department of Chemistry, University of Warwick, Coventry CV4 7AL, U.K.

Ji-Inn Song - Department of Chemistry, University of Warwick, Coventry CV4 7AL, U.K.

Raoul Peltier - Department of Chemistry, University of Warwick, Coventry CV4 7AL, U.K.

Complete contact information is available at:

https://pubs.acs.org/10.1021/acs.biomac.0c01512 


\section{Notes}

The authors declare no competing financial interest.

\section{ACKNOWLEDGMENTS}

We graciously thank the European Research Council (TUSUPO 647106; S.H.E., E.D.H.M., R.P., J.Y.R., and S.P.) and CRUK/EPSRF (Grant no C53561/A19933; C.S.-C.) for financial support. We also acknowledge the STFC for allocation of beam time on SANS-2D (RB1720086) for the characterization of the conjugates and Warwick RTP for access and maintenance to the SEC facilities. The authors would also like to thank Dr Pratik Gurnani and Dr Sarah Rogers for assistance with SANS.

\section{REFERENCES}

(1) Hoffman, A. S. The origins and evolution of "controlled" drug delivery systems. J. Controlled Release 2008, 132, 153-163.

(2) Ahmad, Z.; Shah, A.; Siddiq, M.; Kraatz, H.-B. Polymeric micelles as drug delivery vehicles. RSC Adv. 2014, 4, 17028-17038.

(3) Sercombe, L.; Veerati, T.; Moheimani, F.; Wu, S. Y.; Sood, A. K.; Hua, S. Advances and Challenges of Liposome Assisted Drug Delivery. Front. Pharmacol. 2015, 6, 286.

(4) Li, J.; Mooney, D. J. Designing hydrogels for controlled drug delivery. Nat. Rev. Mater. 2016, 1, 16071.

(5) Zhou, J.; Li, J.; Du, X.; Xu, B. Supramolecular biofunctional materials. Biomaterials 2017, 129, 1-27.

(6) Behzadi, S.; Serpooshan, V.; Tao, W.; Hamaly, M. A.; Alkawareek, M. Y.; Dreaden, E. C.; Brown, D.; Alkilany, A. M.; Farokhzad, O. C.; Mahmoudi, M. Cellular uptake of nanoparticles: journey inside the cell. Chem. Soc. Rev. 2017, 46, 4218-4244.

(7) Champion, J. A.; Katare, Y. K.; Mitragotri, S. Particle Shape: A New Design Parameter for Micro- and Nanoscale Drug Delivery Carriers. J. Controlled Release 2007, 121, 3-9.

(8) Zhao, J.; Lu, H.; Wong, S.; Lu, M.; Xiao, P.; Stenzel, M. H. Influence of nanoparticle shapes on cellular uptake of paclitaxel loaded nanoparticles in 2D and 3D cancer models. Polym. Chem. 2017, 8, 3317-3326.

(9) Truong, N. P.; Whittaker, M. R.; Mak, C. W.; Davis, T. P. The importance of nanoparticle shape in cancer drug delivery. Expert Opin. Drug Delivery 2015, 12, 129-142.

(10) Park, J.-H.; von Maltzahn, G.; Zhang, L.; Schwartz, M. P.; Ruoslahti, E.; Bhatia, S. N.; Sailor, M. J. Magnetic Iron Oxide Nanoworms for Tumor Targeting and Imaging. Adv. Mater. 2008, 20, $1630-1635$.

(11) Müllner, M.; Mehta, D.; Nowell, C. J.; Porter, C. J. H. Passive tumour targeting and extravasation of cylindrical polymer brushes in mouse xenografts. Chem. Commun. 2016, 52, 9121-9124.

(12) Müllner, M.; Yang, K.; Kaur, A.; New, E. J. Aspect-ratiodependent interaction of molecular polymer brushes and multicellular tumour spheroids. Polym. Chem. 2018, 9, 3461-3465.

(13) Dasgupta, S.; Auth, T.; Gompper, G. Shape and Orientation Matter for the Cellular Uptake of Nonspherical Particles. Nano Lett. 2014, 14, 687-693.

(14) Hinde, E.; Thammasiraphop, K.; Duong, H. T. T.; Yeow, J.; Karagoz, B.; Boyer, C.; Gooding, J. J.; Gaus, K. Pair correlation microscopy reveals the role of nanoparticle shape in intracellular transport and site of drug release. Nat. Nanotechnol. 2017, 12, 81-89.

(15) Branco, M. C.; Schneider, J. P. Self-assembling materials for therapeutic delivery. Acta Biomater. 2009, 5, 817-831.

(16) Bong, D. T.; Clark, T. D.; Granja, J. R.; Ghadiri, M. R. SelfAssembling Organic Nanotubes. Angew. Chem., Int. Ed. 2001, 40, 988-1011.

(17) De Santis, P.; Morosetti, S.; Rizzo, R. Conformational Analysis of Regular Enantiomeric Sequences. Macromolecules 1974, 7, 52-58.

(18) Ghadiri, M. R.; Granja, J. R.; Milligan, R. A.; McRee, D. E.; Khazanovich, N. Self-assembling organic nanotubes based on a cyclic peptide architecture. Nature 1993, 366, 324-327.
(19) Couet, J.; Samuel, J. D. J. S.; Kopyshev, A.; Santer, S.; Biesalski, M. Peptide-Polymer Hybrid Nanotubes. Angew. Chem., Int. Ed. 2005, 44, 3297-3301.

(20) Mansfield, E. D. H.; Hartlieb, M.; Catrouillet, S.; Rho, J. Y.; Larnaudie, S. C.; Rogers, S. E.; Sanchis, J.; Brendel, J. C.; Perrier, S. Systematic study of the structural parameters affecting the selfassembly of cyclic peptide-poly(ethylene glycol) conjugates. Soft Matter 2018, 14, 6320-6326.

(21) ten Cate, M. G. J.; Severin, N.; Börner, H. G. Self-Assembling Peptide-Polymer Conjugates Comprising (d-alt-1)-Cyclopeptides as Aggregator Domains. Macromolecules 2006, 39, 7831-7838.

(22) Blunden, B. M.; Chapman, R.; Danial, M.; Lu, H.; Jolliffe, K. A.; Perrier, S.; Stenzel, M. H. Drug Conjugation to Cyclic PeptidePolymer Self-Assembling Nanotubes. Chem.-Eur. J. 2014, 20, 12745-12749.

(23) Larnaudie, S. C.; Brendel, J. C.; Romero-Canelón, I.; SanchezCano, C.; Catrouillet, S.; Sanchis, J.; Coverdale, J. P. C.; Song, J.-I.; Habtemariam, A.; Sadler, P. J.; Jolliffe, K. A.; Perrier, S. Cyclic Peptide-Polymer Nanotubes as Efficient and Highly Potent Drug Delivery Systems for Organometallic Anticancer Complexes. Biomacromolecules 2018, 19, 239-247.

(24) Larnaudie, S. C.; Sanchis, J.; Nguyen, T.-H.; Peltier, R.; Catrouillet, S.; Brendel, J. C.; Porter, C. J. H.; Jolliffe, K. A.; Perrier, S. Cyclic peptide-poly(HPMA) nanotubes as drug delivery vectors: In vitro assessment, pharmacokinetics and biodistribution. Biomaterials 2018, 178, 570-582.

(25) Arnold, O.; Bilheux, J. C.; Borreguero, J. M.; Buts, A.; Campbell, S. I.; Chapon, L.; Doucet, M.; Draper, N.; Ferraz Leal, R.; Gigg, M. A.; Lynch, V. E.; Markvardsen, A.; Mikkelson, D. J.; Mikkelson, R. L.; Miller, R.; Palmen, K.; Parker, P.; Passos, G.; Perring, T. G.; Peterson, P. F.; Ren, S.; Reuter, M. A.; Savici, A. T.; Taylor, J. W.; Taylor, R. J.; Tolchenov, R.; Zhou, W.; Zikovsky, J. Mantid-Data analysis and visualization package for neutron scattering and $\mu$ SR experiments. Nucl. Instrum. Methods Phys. Res., Sect. B 2014, 764, 156-166.

(26) Breßler, I.; Kohlbrecher, J.; Thünemann, A. F. SASfit: a tool for small-angle scattering data analysis using a library of analytical expressions. J. Appl. Crystallogr. 2015, 48, 1587-1598.

(27) Zimm, B. H. The Scattering of Light and the Radial Distribution Function of High Polymer Solutions. J. Chem. Phys. 1948, 16, 1093-1099.

(28) Shaikh, H.; Rho, J. Y.; Macdougall, L. J.; Gurnani, P.; Lunn, A. M.; Yang, J.; Huband, S.; Mansfield, E. D. H.; Peltier, R.; Perrier, S. Hydrogel and Organogel Formation by Hierarchical Self-Assembly of Cyclic Peptides Nanotubes. Chem.-Eur. J. 2018, 24, 19066-19074.

(29) D'souza, A. A.; Shegokar, R. Polyethylene glycol (PEG): a versatile polymer for pharmaceutical applications. Expert Opin. Drug Delivery 2016, 13, 1257-1275.

(30) Bauer, M.; Lautenschlaeger, C.; Kempe, K.; Tauhardt, L.; Schubert, U. S.; Fischer, D. Poly(2-ethyl-2-oxazoline) as Alternative for the Stealth Polymer Poly(ethylene glycol): Comparison of in vitro Cytotoxicity and Hemocompatibility. Macromol. Biosci. 2012, 12, 986-998.

(31) Viegas, T. X.; Bentley, M. D.; Harris, J. M.; Fang, Z.; Yoon, K.; Dizman, B.; Weimer, R.; Mero, A.; Pasut, G.; Veronese, F. M. Polyoxazoline: Chemistry, Properties, and Applications in Drug Delivery. Bioconjugate Chem. 2011, 22, 976-986.

(32) Rho, J. Y.; Brendel, J. C.; MacFarlane, L. R.; Mansfield, E. D. H.; Peltier, R.; Rogers, S.; Hartlieb, M.; Perrier, S. Probing the Dynamic Nature of Self-Assembling Cyclic Peptide-Polymer Nanotubes in Solution and in Mammalian Cells. Adv. Funct. Mater. 2018, 28, 1704569 .

(33) Perrier, S. 50th Anniversary Perspective: RAFT Polymerization-A User Guide. Macromolecules 2017, 50, 7433-7447.

(34) Grube, M.; Leiske, M. N.; Schubert, U. S.; Nischang, I. POx as an Alternative to PEG? A Hydrodynamic and Light Scattering Study. Macromolecules 2018, 51, 1905-1916. 
(35) Benoit, H. On the effect of branching and polydispersity on the angular distribution of the light scattered by gaussian coils. J. Polym. Sci. 1953, 11, 507-510.

(36) Wang, Z. G.; Safran, S. A. Size distribution for aggregates of associating polymers. II. Linear packing. J. Chem. Phys. 1988, 89, 5323-5328.

(37) Danial, M.; Tran, C. M.-N.; Jolliffe, K. A.; Perrier, S. Thermal Gating in Lipid Membranes Using Thermoresponsive Cyclic Peptide-Polymer Conjugates. J. Am. Chem. Soc. 2014, 136, 80188026.

(38) Bolte, S.; Cordelières, F. P. A guided tour into subcellular colocalization analysis in light microscopy. J. Microsc. 2006, 224, 213232.

(39) Schindelin, J.; Arganda-Carreras, I.; Frise, E.; Kaynig, V.; Longair, M.; Pietzsch, T.; Preibisch, S.; Rueden, C.; Saalfeld, S.; Schmid, B.; Tinevez, J.-Y.; White, D. J.; Hartenstein, V.; Eliceiri, K.; Tomancak, P.; Cardona, A. Fiji: an open-source platform for biological-image analysis. Nat. Methods 2012, 9, 676.

(40) Lazzari, G.; Couvreur, P.; Mura, S. Multicellular tumor spheroids: a relevant $3 \mathrm{D}$ model for the in vitro preclinical investigation of polymer nanomedicines. Polym. Chem. 2017, 8, 4947-4969.

(41) Nath, S.; Devi, G. R. Three-Dimensional Culture Systems in Cancer Research: Focus on Tumor Spheroid Model. Pharmacol. Ther. 2016, 163, 94-108.

(42) Chariou, P. L.; Lee, K. L.; Pokorski, J. K.; Saidel, G. M.; Steinmetz, N. F. Diffusion and Uptake of Tobacco Mosaic Virus as Therapeutic Carrier in Tumor Tissue: Effect of Nanoparticle Aspect Ratio. J. Phys. Chem. B 2016, 120, 6120-6129.

(43) Lu, H.; Stenzel, M. H. Multicellular Tumor Spheroids (MCTS) as a 3D In Vitro Evaluation Tool of Nanoparticles. Small 2018, 14, 1702858. 